\title{
La interrupción legal del embarazo en Argentina
}

\author{
The legal termination of pregnancy in Argentina
}

Vilda Discacciati ${ }^{\mathrm{a}, \mathrm{b}, \mathrm{c}}$, Daniela Epstein ${ }^{\mathrm{a}}$, Nadia Musarella $^{\mathrm{a}, \mathrm{d}}$, Sergio Terrasa ${ }^{\mathrm{a}, \mathrm{e}, \mathrm{c}}$, Camila Volij $^{\mathrm{a}}$, Mercedes Volpi $^{\mathrm{a}, \mathrm{d}}$

\begin{abstract}
Resumen
En el contexto de la publicación por parte del Ministerio de Salud de la Nación de Argentina del Protocolo para la atención integral de las personas con derecho a la interrupción legal del embarazo (ILE), resumido y comentado en este mismo número de EVIDENCIA, este comentario editorial ofrece: 1) una perspectiva amplia de lo que significa el aborto, desde la mirada estrictamente biologicista hasta definiciones basadas en el enfoque de género; 2) una descripción de los diferentes marcos jurídicos respecto del aborto que imperan actualmente en Latinoamérica y Argentina, haciendo especial hincapié en lo concerniente a las ILE; 3) información epidemiológica sobre la mortalidad materna en Argentina y la asociada al aborto inseguro; 4) estadísticas sobre la realización de ILE en la Ciudad Autónoma de Buenos Aires, Argentina.

Dada la situación actual de aislamiento social preventivo obligatorio en el marco de la pandemia de Covid-19, consideramos quienes integramos equipos de salud debemos estar más atentos/as que nunca a las situaciones de violencia potencial que podrían suceder en este contexto, para dar las respuestas pertinentes -entre las que se incluyen las ILE-, a las víctimas de embarazos no deseados consecutivos a situaciones de coerción.
\end{abstract}

\begin{abstract}
In the context of the publication by the Argentine Ministry of Health of the Protocol for the comprehensive care of people with the right to legal termination of pregnancy (LTP), summarized and commented on in this same issue of EVIDENCIA, this editorial article offers: 1) a broad perspective of what abortion means, from a strictly biological point of view to definitions based on the gender approach; 2) a description of the different legal frameworks regarding abortion that currently prevail in Latin America and Argentina, with special emphasis on LTP; 3) epidemiological information on maternal mortality in Argentina and that associated with unsafe abortion; 4) statistics on the realization of LTP in the Autonomous City of Buenos Aires, Argentina.

Given the current situation of mandatory preventive social isolation in the framework of the Covid-19 pandemic, we consider that those of us who are part of health teams should be more alert than ever to situations of potential violence that could occur in this context, to give the relevant responses - including LTP- to victims of unwanted pregnancies following coercive situations.
\end{abstract}

Palabras clave: Aborto Legal, Aborto Inducido, Aborto, Violación, Violencia Doméstica, Violencia contra la Mujer, Delitos Sexuales. Keywords: Legal Abortion, Induced Abortion, Abortion, Rape, Domestic Violence, Violence Against Women, Sex Offenses.

Discacciati V, Epstein D, Musarella N, Terrasa S, Volij C y Volpi M.. La interrupción legal del embarazo en Argentina. Evid Actual Pract Ambul. 2020;23(1):e002050.

\section{Introducción}

Desde una perspectiva biologicista se define al aborto como la interrupción voluntaria o involuntaria del embarazo antes de que el embrión o feto esté en condiciones de vivir fuera del vientre materno.

Desde el marco jurídico de cada país, el aborto puede ser legal o ilegal.

Por otro lado, dependiendo de las condiciones en las cuales se lleve a cabo el aborto, este puede ser sin riesgo o inseguro. La Organización Mundial de la Salud (OMS) define al aborto inseguro como "un procedimiento para finalizar un embarazo no deseado realizado por personas que carecen de la capacidad necesaria o que se lleva a cabo en un entorno donde se carece de un estándar médico mínimo, o ambos" 1 .

Si bien estas definiciones son las mayormente conocidas y utilizadas en los diversos ámbitos sociales, marcan un recorte muy acotado de la realidad y conducen a un análisis en principio superficial y limitado de las problemáticas vinculadas a la temática. Al ampliar el concepto desde otras perspectivas sociales, se pueden manifestar otras cuestiones asociadas muy relevantes.
Recordemos que el ámbito sanitario se inscribe en un marco que parte de la definición de salud: "estado de completo bienestar físico, mental y social, y no solamente la ausencia de afecciones o enfermedades" (según la definición de la OMS). Este concepto marca una complejidad en sí misma, en un campo en el que interactúan múltiples factores sociales, políticos, económicos, culturales y científicos ${ }^{2}$. Por ende, la salud implica una multiplicidad de procesos dentro de un momento histórico, ya que cada contexto histórico maneja cierta estructura de poder, normas, valores, técnicas y creencias, por lo que la noción de salud resulta en una construcción social.

\section{La violencia}

Desde una perspectiva de género, el aborto ${ }^{3}$ se plantea como un hecho social denso y complejo, en el contexto de relaciones de poder donde están involucradas la salud, la sexualidad, la moral y la ética, e incluso el significado mismo de la maternidad. Por lo tanto, según el enfoque del que se parta para definir aborto, se permitirá visibilizar o no la trama de violencias simbólicas y físicas que atraviesan las personas con capacidad de gestar.

\footnotetext{
a Servicio de Medicina Familiar y Comunitaria, Hospital Italiano de Buenos Aires. daniela.epstein@hospitalitaliano.org.ar, camila.volij@hospitalitaliano.org.ar,

b Comité de Bioética, Hospital Italiano de Buenos Aires.

c Departamento de Salud Pública, Instituto Universitario Hospital Italiano de Buenos Aires. vilda.discacciati@hospitalitaliano.org.ar, sergio.terrasa@hospitalitaliano.org.ar

d Departamento de Medicina, Instituto Universitario Hospital Italiano de Buenos Aires. nadia.musarella@hospitalitaliano.org.ar, mercedes.volpi@hospitalitaliano.org.ar

e Departamento de Investigación, Hospital Italiano de Buenos Aires.
} 
Siguiendo este enfoque, la violencia es un eje relevante a destacar sobre la temática del aborto. La violencia es un problema central que involucra a la sociedad por completo y que requiere del compromiso de diferentes sectores, instituciones y organizaciones -tanto de gestión pública como privada-, para reflexionar y diseñar cursos de acción que permitan mitigarla.

Dentro de la esfera sanitaria, la violencia (entendiéndola como una asimetría de poder), se visibiliza cuando se la pesquisa, es decir, cuando se la busca o interroga, cuando la consulta lleva a pensarla como opción diagnóstica, o cuando es explícita en un pedido de ayuda de una persona que está sufriendo una situación grave de salud. Por este motivo, se ha convertido en una luz de alarma que abre el interrogante sobre cómo se está trabajando en los equipos de salud a fin de diseñar estrategias de prevención y abordaje.

Esta línea de pensamiento permite comprender las diferentes modalidades y formas en las cuales se cristaliza la violencia en el ámbito sanitario. Al revisar las estadísticas nacionales, y particularmente las referidas a la salud reproductiva, los datos llamativos son la tasa de fecundidad adolescente y mortalidad materna (ver más adelante), donde el abuso y la violencia de género permiten entrever cómo se ve afectada la salud de mujeres (y personas gestantes) en el recorte de la salud sexual ${ }^{4-7}$. Esto se suma al eje de la inequidad de género $y$, al mismo tiempo, da cuenta de cómo se ve limitado el acceso a la salud en mujeres y población trans $^{8}$.

Otro punto a considerar son los embarazos forzados. El Fondo de Naciones Unidas para la Infancia (UNICEF) informó que, en Argentina, alrededor de 2500 niñas atraviesan un parto cada año ${ }^{9}$, definiendo como embarazo forzado al que ocurre y se mantiene sin la voluntad de la persona gestante, y considerándolo como uno de los efectos posibles de los abusos sexuales. Cabe destacar que la mayoría de los embarazos de niñas menores de 15 años son producto de violencia ${ }^{10}$. Es por este motivo que todas las niñas y adolescentes menores de 15 años tienen el derecho de solicitar una interrupción legal del embarazo (ILE), y si la gestación fue producto de violencia sexual, de acceder también a atención psicosocial, a medidas de protección e intervención de la justicia.

Es probable que la problemática de los embarazos forzados resulte más tangible en niñas y adolescentes que en las personas adultas, pero cabe resaltar que cuando ocurren relaciones sexuales no consentidas en el contexto de un matrimonio, esta situación también se trata de violencia doméstica y de pareja. Garantizar el derecho a la ILE forma parte de la práctica médica para cuidar la salud de la persona con capacidad de gestar. Por el contrario, obstaculizar su acceso, implicaría una vulneración de derechos y un hecho de violencia hacia una persona que ya se encuentra en una situación vulnerable.

\section{Modelos jurídicos vigentes en Latinoamérica para regular la interrupción del embarazo}

La situación jurídica del aborto es un importante indicador de la accesibilidad que tienen las mujeres a la posibilidad de ejercer sus derechos reproductivos. Existen diferentes modelos jurídicos a nivel mundial: interrupción del embarazo legal sin restricciones, dentro de plazos gestacionales establecidos, como en Uruguay y Cuba; ilegal, sin excepciones, como en Nicaragua, Honduras y E Salvador; o bien, legal en algunas circunstancias determinadas, como en Argentina, Bolivia y Perú ${ }^{11}$

\section{Reseña histórica y situación legal actual del aborto en Argentina}

En Argentina, desde 1921, el artículo 86 del Código Penal de la Nación establece excepciones a la punibilidad del aborto: inciso $1^{\circ}$ ) en caso de peligro para la vida o la salud de la madre y si este peligro no puede ser evitado por otros medios; inciso $2^{\circ}$ ) si el embarazo proviene de una violación o de un atentado al pudor cometido sobre una mujer idiota o demente ${ }^{12}$.

Sin embargo, la implementación de esta legislación se vio entorpecida por múltiples motivos. Un hecho histórico que tuvo como objetivo revertir la falta de incumplimiento del artículo mencionado, fue el dictamen del "fallo FAL" en 2012, que estableció que toda mujer víctima de violación no puede ni debe ser obligada a solicitar una autorización judicial para interrumpir su embarazo ${ }^{13}$.

Otro intento de fomentar el cumplimiento de la ley fue en 2015 , con la publicación del "Protocolo para la atención integral de las personas con derecho a la interrupción legal del embarazo" ${ }^{14}$, Este documento fue elaborado por el Ministerio de Salud de la Nación, con información médica, bioética y legal para la implementación de la ILE. Sin embargo, a pesar de establecer que el protocolo "...es de aplicación obligatoria en todo el territorio argentino y debe ser puesto en práctica por todas las instituciones sanitarias, tanto públicas como privadas...", carecía al momento de su publicación de estatus de resolución ministerial.

Hasta abril de 2017, apenas ocho de las 25 jurisdicciones de todo el país habían adherido al protocolo nacional mencionado o contaban con protocolos propios, con buen ajuste a los criterios establecidos por la Corte Suprema de Justicia de la Nación (CSJN). Otras ocho provincias habían elaborado protocolos incluyendo requisitos que dificultan, en vez de facilitar el acceso de mujeres a servicios de aborto seguro -a los que tienen derecho, de acuerdo a la legislación de orden superior ya mencionada-, y las ocho jurisdicciones restantes, no contaban con normas locales ${ }^{15}$ (verFigura 1).

Teniendo en cuenta las diversas problemáticas a nivel nacional para la implementación de la ILE, en 2018 se presentó un proyecto de ley en el Congreso de la Nación para la interrupción voluntaria del embarazo (IVE). En Junio de ese año el proyecto fue aprobado por la Cámara de Diputados y dos meses después, rechazado por la Cámara de Senadores. En consecuencia, el marco legal vigente no se modificó ese año.

Sin embargo, el debate sobre el aborto legal mantuvo presente y activo en la opinión pública, en los medios de comunicación, en las redes sociales ${ }^{16}$, en la comunidad médica, y en organismos gubernamentales y sanitarios. La confrontación ideológica a nivel gubernamental se hizo evidente cuando, en noviembre de 2019, fue derogada a través del decreto nacional 785/2019 publicado en el boletín oficial del Ministerio de Justicia y Derechos Humanos ${ }^{17}$ la resolución 3158/2019 de la Secretaría de Gobierno de Salud que acababa de actualizar el "Protocolo para la atención integral de las personas con derecho a la interrupción legal del embarazo" ${ }^{18}$, justo al día siguiente de su publicación.

Finalmente, con la asunción de nuevas autoridades nacionales en diciembre de 2019, el Ministerio de Salud de la Nación Argentina repuso la resolución ${ }^{19}$, publicando el protocolo vigente (resumido y comentado en este mismo número de EVIDENCIA ${ }^{20}$ ), el cual afirma que los principios generales para la atención de la ILE son: el respeto de la autonomía, la no judicialización, la accesibilidad, la confidencialidad, la privacidad, la celeridad y la transparencia activa ${ }^{21}$ 
Figura 1. Mapa de Argentina con las distintas jurisdicciones y sus respectivos protocolos para la interrupción legal del embarazo (2015). Fuente: Amnistía Internacional. El estado de situación de la interrupción legal del embarazo. El marco legal y las violaciones a los derechos humanos detrás de los obstáculos al aborto legal, 2017.

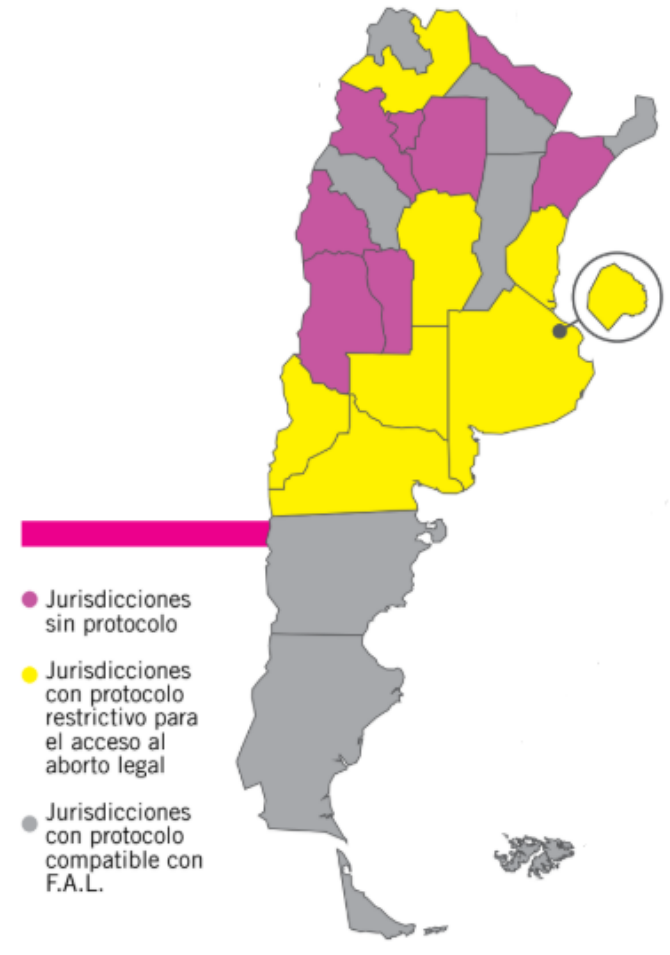

\section{Estadísticas del aborto en Argentina}

Como mencionamos al principio de este artículo, desde la mirada jurídica el aborto puede ser legal o ilegal; pero desde otro mar$\mathrm{co}$, puede ser seguro o inseguro. Estos conceptos van de la mano, ya que en los países donde la interrupcion del embarazo es legal se ofrecen abortos sin riesgos a todas las personas que lo solicitan, mientras que en los países donde esta práctica es ilegal o en los que presentan una restricción legal (como Argentina), suele darse un acceso desigual al aborto sin riesgos. En estos contextos, los abortos que cumplen con los requisitos de seguridad se convierten en el privilegio de las personas que pueden pagar para acceder a realizarlos, mientras que las mujeres de escasos recursos económicos no tienen otra opción que someterse a abortos inseguros que provocan discapacidades y muerte.

Se denomina muerte materna a la de una mujer durante el embarazo o durante los 42 días posteriores a su terminación, independientemente de la duración y localización de la gestacion, por cualquier causa relacionada o agravada por el embarazo o su atención, pero no por causas accidentales o incidentales. Por este motivo la muerte materna es uno de los indicadores más dramáticos de las inequidades en salud, ya que afecta desproporcionadamente a las mujeres más pobres y vulnerables, para quienes el embarazo o la maternidad terminan convirtiéndose en un riesgo desmesurado para su vida y su salud ${ }^{22}$.

La estructura causal de las muertes maternas en Argentina ha variado poco en las últimas dos décadas. Las complicaciones relacionadas con el aborto representan, al igual que en Jamaica y Trinidad Tobago, el $30 \%$ del total de muertes maternas ${ }^{23}$. Entre 2004 y 2008, estas complicaciones fueron la causa principal en 14 de las 24 provincias de Argentina, y la segunda causa en otras cinco.

Si bien se trata de estimaciones indirectas, en Argentina ocurrirían entre 370 y 522 mil interrupciones de embarazos por año ${ }^{24}$. Esta información proviene del número de hospitalizaciones producidas en los establecimientos públicos por complicaciones relacionadas con abortos, sin distinguir entre los espontáneos y los provocados $^{25}$.

Figura 2. Mapa de Argentina con las distintas jurisdicciones y sus respectivos protocolos para la interrupción legal del embarazo (2020). Fuente: elaboración propia.

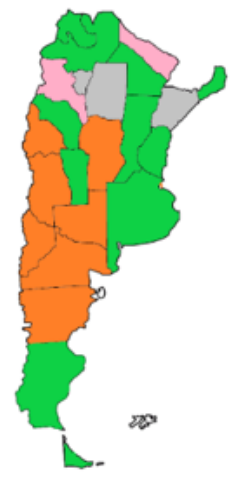

\begin{tabular}{|l|l|}
\hline & $\begin{array}{l}\text { Jurisdicciones adheridas } \\
\text { propio }\end{array}$ \\
\hline $\begin{array}{l}\text { Jurisdicciones con protocolo } \\
\text { que utilizan protocolo }\end{array}$ \\
\hline $\begin{array}{l}\text { Jurisdicciones no adheridas } \\
\text { sin protocolo }\end{array}$ \\
\hline
\end{tabular}




\section{Datos sobre interrupciones legales del embarazo en Ciudad Autónoma de Buenos Aires}

En la Ciudad Autónoma de Buenos Aires (CABA), el Programa de Salud Sexual y Reproductiva del Ministerio de Salud se fundamenta en los derechos, el género y el acceso universal a las prestaciones que garanticen los cuidados, tomado como uno de los principales basamentos filosóficos de la salud sexual y reproductiva, el derecho individual a disfrutar (o al menos, no padecer) el ejercicio de la sexualidad.

El último boletín (12/2019) de ese programa indica que de las niñas que tuvieron su primer embarazo entre los 12 y los 14 años, el $76,3 \%$ no lo buscó. Este dato sugiere un inicio de relaciones sexuales no deseadas y/o físicamente forzadas.

Por otro lado, los datos sobre el acceso a la ILE muestran que este ha ido en aumento entre 2014 y 2018. En 2014, nueve establecimientos -ocho hospitales y un Centro de Salud Comunitaria (CeSAC)- comunicaron por lo menos una ILE. En 2018, el número total de establecimientos alcanzó los 53 (39 CeSAC y 14 hospitales). Las mujeres que accedieron a la ILE durante 2018 tenían una edad promedio de 26 años, mientras que $16 \%$ de las situaciones ocurrieron en adolescentes. El causal salud fue aquel que se registró con mayor frecuencia, seguido por el causal violación, ocupando el tercer lugar el riesgo para la vida materna, y el cuarto, un feto incompatible con la vida 26

No se dispone de datos publicados del sector privado de salud.

\section{A modo de conclusiones}

A partir del impulso de los colectivos de mujeres y los feminismos, que en voz alta han puesto estos temas en la agenda política, vienen presentándose desde 2007 proyectos de ley que pretenden garantizar a nivel nacional el derecho al aborto legal seguro y gratuito ${ }^{27}$. Desde 2015 -con el inicio en Argentina del movimiento "Ni una menos"- y desde 2018, con el comienzo del debate a nivel nacional sobre la legalización/despenalización del aborto, han ganado visibilidad las inequidades de género presentes en nuestra sociedad y han comenzado a empoderarse -con resultados todavía parciales- las mujeres y otros colectivos a los que pertenecen la mayoría de las víctimas de violencia institucional y/o sanitaria a las que nos hemos referido durante este artículo.

Como ya afirmara en 2018 una de las autoras de este artícu$10^{28}$, en Argentina "... el aborto ha salido de armario, pero nó de la clandestinidad". Si bien las personas que firmamos este comentario editorial, consideramos que están dadas las condiciones políticas para que el aborto comience realmente a salir de la clandestinidad en Argentina ${ }^{29}$, es probable que los actuales sucesos enmarcados en la pandemia de la enfermedad por Covid-19, retrasen este proceso.

Por esta razón, durante un tiempo, quienes trabajamos en el Sistema de Salud nos veremos obligados a continuar respaldándonos en la actual legislación sobre ILE y en sus protocolos de implementación, para ayudar a las víctimas de embarazos no deseados. Además, consideramos que desde el equipo de salud debemos estar más atentos que nunca a las situaciones de violencia potencial ${ }^{30}$ que podrían suceder en este contexto de aislamiento domiciliario obligatorio para dar las respuestas pertinentes, entre las que se incluyen las ILE.

Para finalizar, quienes firmamos este artículo deseamos fervientemente que estas últimas conclusiones se vuelvan obsoletas en el menor tiempo posible.

\section{Referencias}

1. Organización Mundial de la Salud, Departamento de Salud Reproductiva e Investigaciones Conexas. Aborto sin riesgos: guía técnica y de políticas para sistemas de salud. 2nd ed. Organización Mundial de la Salud; 2012.

2. Briceño-León R, De-Souza M, Coimbra C. Bienestar, Salud Pública y Cambio Social. In: Salud y equidad: una mirada desde las ciencias sociales Rio de Janeiro, Brazil: Editora Fio-Cruz; 2000. p. 15-24.

3. Gamba S, Barrancos D, Giberti E, Maffia D. Diccionario de estudios de género y feminismos. 2nd ed. Biblos; 2009.

4. García-Moreno C, , et al. Estudio multipaís de la OMS sobre salud de la mujer y la violencia doméstica : primeros resultados sobre eventos relativos a la salud y respuestas de las mujeres a dicha violencia: resumen del informe prevalencia,. Ginebra, Suiza: Organización Mundial de la Salud; 2005.

5. Campbell JC, García-Moreno C, Sharps PW. Abuse During Pregnancy in Industrialized and Developing Countries. Violence Against Women. 2004;10(7):770-789. Available from: 10.1177/1077801204265551;https://dx.doi.org/10.1177/1077801204265551.

6. Ellsberg M, Jansen H, Heise L, et al. Intimate partner violence and women's physical and mental health in the WHO multi-country study on women's health and domestic violence: an observational study. LANCET. 2008;371(9619):1165-1172. Available from: 10.1016/S0140-6736(08)60522-X.

7. Bott S, Guedes A, Goodwin M, et al. Violencia contra la mujer en América Latina y el Caribe: análisis comparativo de datos poblacionales de 12 países. and others, editor. Organización Panamericana de la Salud. Centros para el Control y la Prevención de Enfermedades de los Estados Unidos.; 2014.

8. Global and regional estimates of violence against women: prevalence and health effects of intimate partner violence and nonpartner sexual violence. Ginebra, Suiza: Organización Mundial de la Salud; 2013. Available from: https://apps.who.int/iris/bitstream/handle/10665/85239/9789241564625_ eng.pdf?sequence $=1$.

9. Plan Nacional de Prevención del Embarazo No Intencional en la Adolescencia. Acceso a la justicia: abusos sexuales y embarazos forzados en niñas y adolescentes menores de 15 años Documento técnico No 6 Noviembre; 2019. Available from: https://www.unicef.org/argentina/media/7211/file/ Acceso\%20a\%20la\%20Justicia.pdf.

10. Abusos sexuales y embarazo forzado en la niñez y adolescencia: lineamientos para su abordaje interinstitucional (dirigido por Silvia Chejter); 2018.

11. Johnson BR, Mishra V, Lavelanet AF, et al. A global database of abortion laws, policies, health standards and guidelines. Bull World Health Organ. 2017;95(7):542-544. Available from: 0.2471/BLT.17.197442.

12. Argentina Ministerio de Justicia y Derechos Humanos. Código Penal de la Nación Argentina. Ley 11.179. Delitos contra las personas;. Available from: http://servicios.infoleg.gov.ar/infoleglnternet/anexos/15-19999/16546/texact.htm\#15.

13. Argentina Corte Suprema de Justicia de la Nación. F., A. L. s/ Medida autosatisfactiva; $2012 . \quad$ Available from: http://www.saij.gob.ar/cortesuprema-justicia-nacion-federal-ciudad-autonoma-buenos-aires--medida-autosatisfactiva-fa12000021-2012-03-13/123456789-120-0002-1 otseupmocsollaf.

14. Argentina Ministerio de Salud. Protocolo para la atención integral de las personas con derecho a la interrupción legal del embarazo. 2 nd ed.; 2015. Available from: http://www.msal.gob.ar/images/stories/bes/graficos/0000000875cnt-protocolo_ile_octubre\%202016.pdf.

15. Amnistía Internacional. El estado de situación de la interrupción legal del embarazo. El marco legal y las violaciones a los derechos humanos detrás de los obstáculos al aborto legal; 2017. Available from: https://amnistia.org.ar/wp-content/uploads/delightful-downloads/2017/10/01-informe-estadoILE-Mapa-actualizado-2.pdf [Last access: 2020-03-30].

16. Amnistía Internacional. Pañuelos Verdes. Relatos de la violencia durante el debate por la legalización de la interrupción legal del embarazo; 2018. Available from: https://amnistia.org.ar/wp-content/uploads/delightful-downloads/2018/12/PA\%C3\%91UELOS-VERDES-entrega02-online-FINAL.pdf [Last access: 2020-03-30].

17. Argentina, Ministerio de Justicia y Derechos Humanos. Decreto nacional 785/2019. Derogación de la Resolución de la Secretaría de Gobierno de Salud 3158/2019 que aprobó el Protocolo para la Atención Integral de las Personas con Derecho a la Interrupción Legal del Embarazo ; 2019. 
Available from: http:/www.saij.gob.ar/785-nacional-derogacion-resolucion-secretaria-gobierno-salud-3158-2019-aprobo-protocolo-para-atencionintegral-personas-derecho-interrupcion-legal-embarazo-dn20190000785-2019-11-21/123456789-0abc-587-0000-9102soterced?\&0=1\&f=Total\% 7CFecha\%7CEstado\%20de\%20Vigencia/Vigente\%2C\%20de\%20alcance\%20general\%7CTema\%5B5\%2C1\%5D\%7COrganismo\%5B5\%2C1\% 5D\%7CAutor\%5B5\%2C1\%5D\%7CJurisdicci\%F3n/Nacional\%7CTribunal\%5B5\%2C1\%5D\%7CPublicaci\%F3n\%5B5\%2C1\%5D\%7CColecci\%F3n\% 20tem\%E1tica\%5B5\%2C1\%5D\%7CTipo\%20de\%20Documento/Legislaci\%F3n/Decreto\&t=9285.

18. Argentina Ministerio De Salud Y Desarrollo Social Secretaría De Gobierno De Salud . Resolución 3158/2019; 2019. Available from: https://www. boletinoficial.gob.ar/detalleAviso/primera/221438/20191120.

19. Argentina Ministerio de Salud. Resolución 1/2019; 2019. Available from: https://www.boletinoficial.gob.ar/detalleAviso/primera/223829/20191213.

20. Franco JVA, Volij C. Nuevo protocolo para la interrupción legal del embarazo en Argentina. Comentado de: Ministerio de Salud. Resolución 1/2019. Protocolo para la atención integral de las personas con derecho a la interrupción legal del embarazo. Boletín Oficial de la República Argentina 12/12/2019. Evid Actual Pract Ambul. 2019;23(1):e002029.

21. Argentina Ministerio de Salud. Protocolo para la atención integral de las personas con derecho a la interrupción legal del embarazo. Segunda Edición.; 2019.

22. Romero M, Chapman E, Ramos S, et al. La situación de la mortalidad materna en la Argentina. Hoja informativa Observatorio de Salud Sexual y Reproductiva. 2010;1. Available from: http://www.ossyr.org.ar/pdf/hojas informativas/Hoja Informativa OSSyR 01.pdf.

23. González-Vélez AC. Una mirada analítica a la legislación sobre interrupción del embarazo en países de Iberoamérica y el Caribe. Comisión Económica para América Latina (CEPAL); 2011. Available from: https://www.cepal.org/es/publicaciones/5837-mirada-analitica-la-legislacion-interrupcionembarazo-paises-iberoamerica-caribe.

24. Mario S, Pantelides EA. Estimación de la magnitud del aborto inducido en la Argentina. Comisión Económica para América Latina (CEPAL); 2009. Available from: https://repositorio.cepal.org/handle/11362/12842.

25. Argentina Dirección de Estadísticas e Información de Salud, Ministerio de Salud de la Nación. Estadísticas vitales. Información básica, año 2015; 2015.

26. Portnoy F. Salud sexual y reproductiva en la ciudad de Buenos Aires. Situación y respuesta 2016-2018. Ciudad Autónoma de Buenos Aires. Ministerio de Salud. Coordinación Salud Sexual, Sida e ITS; 2019. Available from: https://www.buenosaires.gob.ar/salud/sexual-y-vih/datos-oficiales-saludsexual-caba.

27. Campaña Nacional por el Derecho al Aborto Legal Seguro y Gratuito;. Available from: http://www.abortolegal.com.ar/about/ [Last access: 2020-0330].

28. Discacciati V. En Argentina, durante 2018 el aborto salió del armario pero no de la clandestinidad. Evid Actual Pract Ambul. 2018;21(2):42-44. Available from: http://www.evidencia.org.ar/index.php/Evidencia/article/view/3316.

29. Dillon M. Pañuelazo por el aborto: Este es el año, en 2020 será ley; 2020. Available from: https://www.pagina12.com.ar/248531-panuelazo-por-elaborto-este-es-el-ano-en-2020-sera-ley [Last access: 2020-03-30].

30. Fernández-Escudero C. Violencia de género: hubo un $30 \%$ más de llamadas por el aislamiento. Coronavirus. Pandemia de Coronavirus.; 2020. Available from: https://www.perfil.com/noticias/coronavirus/violencia-de-genero-30-mas-de-llamadas-por-el-aislamiento.phtml [Last access: 202003-30]. 